\title{
Assessment of the LDL-C/HDL-C ratio as a predictor of one year clinical outcomes in patients with acute coronary syndromes after percutaneous coronary intervention and drug-eluting stent implantation
}

Zhixiong Zhong ${ }^{1,3,4,5,7,8+}$, Jingyuan $\mathrm{Hou}^{2,3,4,5,6,7,8+}$, Qifeng Zhang ${ }^{1,3,4,5,7}$, Wei Zhong ${ }^{1,3,4,5,7}$, Bin $\mathrm{Li}^{1,3,4,5,7}$, Cunren $\mathrm{Li}^{1,3,4,5,7}$, Zhidong Liu ${ }^{1,3,4,5,7}$, Min Yang ${ }^{1,3,4,5,7}$ and Pingsen Zhao ${ }^{2,3,4,5,6,7,8^{*}}$ (D)

\begin{abstract}
Background: Despite significant advances in the management of acute coronary syndromes (ACS), there are still plenty of patients undergoing percutaneous coronary intervention (PCI) and stent implantation suffered poor prognosis and high treatment expenditure. Evidence increasingly suggests that the ratio of low-density lipoprotein cholesterol/high-density lipoprotein cholesterol (LDL-C/HDL-C) ratio might be a novel marker for the risk of atherosclerotic cardiovascular disease, but the impact of LDL-C/HDL-C ratio on 1-year prognosis of drug-eluting stent (DES) implantation patients after $\mathrm{PCl}$ is still not reported. Our aim of the study was to investigate the impact of LDL-C/HDL-C ratio on 1-year prognosis of DES implantation patients after PCl.
\end{abstract}

Methods: Between May 2014 and July 2016, 1937 patients who were underwent primary PCI and DES implantation and achieving LDL-C with statins were enrolled and divided into two groups based on the ratio of LDL-C/HDL-C.

Results: The entire occurrence of adverse cardiovascular events according to the ratio of LDL-C/HDL-C showed that there were no significant differences in 1-year cardiovascular death (hazard ratio [HR]: 1.97, 95\% confidence interval [Cl]: 0.49 to $7.84, P=0.329$ ), myocardial infarction (MI) (HR: 1.66, 95\% Cl: 0.84 to $3.28, P=0.172)$ and bleeding events (HR: $1.08,95 \%$ Cl: 0.83 to $1.41, P=0.598$ ) The cumulative incidence of target lesion revascularization (TLR) (HR: 1.43, 95\% Cl: 1.10 to $1.86, P=0.007$ ), stent thrombosis (ST) (HR: 2.04, 95\% Cl: 1.06 to 3.93, $P=0.037$ ) and major adverse cardiac events (MACE) (HR: $1.54,95 \%$ Cl: 1.24 to $1.91, P<0.001$ ) were significantly higher in high group than in low group. Multivariate Cox regression analysis revealed that age (HR: 1.556, 95\%, Cl: 1.198 to 2.021, $P<0.001$ ), together with diabetes mellitus (HR: 1.490, 95\% Cl: 1.142 to $1.945, P=0.003$ ), and ratio of LDL-C/HDL-C (HR: 1.638, 95\% Cl: 1.260 to 2.218, $P<0.001$ ) were independent predictors of 1-year MACE. The Kaplan-Meier cumulative MACE-free survival curves with a log-rank test showed that the presence of high ratio of LDL-C/HDL-C was associated with higher incidences of MACE after PCI with DES implantation.

(Continued on next page)

\footnotetext{
* Correspondence: zhaopingsen01@163.com; zhaopingsen@hotmail.com ${ }^{\dagger}$ Zhixiong Zhong and Jingyuan Hou contributed equally to this work. ${ }^{2}$ Clinical Core Laboratory, Meizhou People's Hospital (Huangtang Hospital), Meizhou Academy of Medical Sciences, Meizhou Hospital Affiliated to Sun Yat-sen University, Meizhou 514031, People's Republic of China

${ }^{3}$ Center for Precision Medicine, Meizhou People's Hospital (Huangtang Hospital), Meizhou Academy of Medical Sciences, Meizhou Hospital Affiliated to Sun Yat-sen University, Meizhou 514031, People's Republic of China Full list of author information is available at the end of the article
}

(c) The Author(s). 2019 Open Access This article is distributed under the terms of the Creative Commons Attribution 4.0 International License (http://creativecommons.org/licenses/by/4.0/), which permits unrestricted use, distribution, and reproduction in any medium, provided you give appropriate credit to the original author(s) and the source, provide a link to the Creative Commons license, and indicate if changes were made. The Creative Commons Public Domain Dedication waiver (http://creativecommons.org/publicdomain/zero/1.0/) applies to the data made available in this article, unless otherwise stated. 
(Continued from previous page)
Conclusions: The high LDL-C/HDL-C ratio was associated with cardiovascular events in patients with ACS after PCl and
DES implantation.

Keywords: Acute coronary syndromes, Percutaneous coronary intervention, Drug-eluting stent implantation, Low-density lipoprotein, High-density lipoprotein, Major adverse cardiac events

\section{Background}

Cardiovascular diseases (CAD) is a significant global health problem and is considered as the leading cause of death worldwide [1]. Currently, approximately 46 million people in China are affected by CAD with the changes lifestyle and longer life spans [2]. With the introduced of coronary artery bypass graft (CABG) surgery and percutaneous coronary intervention (PCI), substantial breakthrough have been made in the treatment of CAD, especially in patients with acute coronary syndromes (ACS) [3, 4]. In particular, the advent of drug-eluting stent (DES) has significantly reduced the occurrence of vessel revascularization and major adverse cardiac events (MACE) [5, 6]. Despite significant advances in the management of ACS, there are still plenty of patients undergoing PCI and stent implantation suffered poor prognosis and high treatment expenditure attribute to the varying disease severity $[7,8]$. Therefore, identifying cardiovascular risk factors that affect the prognosis of patients and implementation of treatments to minimize risk of new ischemic events after PCI treatment is necessary [9-12].

Previous literature has illustrated that dyslipidemia is a risk factor for development and progression of coronary arteriosclerosis and cardiovascular outcomes in patients with CAD [13, 14]. Our previous research confirmed that serum lipid levels varied in age and gender in Hakka patients with acute myocardial infarction (AMI). Dyslipidemia is more prevalent in the non-elderly than in the elderly for males. Levels of total cholesterol (TC), triglycerides (TG) and low-density lipoprotein cholesterol (LDL-C) were higher in females than in males for the elderly Hakka population in southern China [15]. Numerous evidences have revealed that LDL-C serum concentrations correlate with very high cardiovascular risk and stringent LDL-C lowering with statins therapy is recommended for the secondary prevention of recurrent cardiovascular events and survival improvement after PCI by the current international guidelines [16, 17]. Another research of ours found that PCI patients with lower preprocedural LDL-C were less risky to periprocedural myocardial injury in southern China [18]. Data from patients treated with statins show considerable gains by approximately $22 \%$ per $1.0 \mathrm{mmol} / \mathrm{L}$ LDL-C reduction [19]. However, many patients achieving very low levels of LDL-C showing widely different treatment effects and subsequently experienced a cardiovascular event because of the residual risk factors [11, 13]. Recently, serum levels of high-density lipoprotein cholesterol (HDL-C) was recognized significantly and inversely associated with the development of atherosclerotic cardiovascular diseases [20,21]. Evidence increasingly suggests that the ratio of LDL-C/HDL-C ratio might be a novel marker of the risk of atherosclerotic cardiovascular disease, as it simultaneously evaluates the levels of both LDL-C and HDL-C [22, 23]. However, there is little information regarding the predictive value of the LDL-C/HDL-C ratio for detecting cardiovascular events in patients with ACS after DES implantation and more studies are needed to further characterize the relationship between the LDL-C/HDL-C ratio and clinical outcome. The purpose of this study was to investigate the impact of LDL-C/HDL-C ratio on 1-year prognosis of DES implantation patients after PCI.

\section{Materials and methods Study population}

This was a retrospective observational study. Between May 2014 and July 2016, a total of 1937 consecutive participants who had the initiation of statins therapy with atorvastatin after successful primary PCI were enrolled from the patients who had been hospitalized at department of cardiology in the Meizhou People's Hospital. All patients had measurements of serum lipid profiles at baseline and at 4 weeks after atorvastatin therapy and lipid levels were found to remain constant during 1-year follow up. The study protocol was approved by the Ethics Committee of Meizhou People's Hospital (Huangtang Hospital), Meizhou Academy of Medical Sciences, Meizhou Hospital Affiliated to Sun Yat-sen University, Guangdong province, China, and conforms to the ethical principles of the Declaration of Helsinki. All participants have signed the written informed consents.

Patients were eligible for enrollment if (1) if they were18 years of age or older; (2) if they were atorvastatin-treated who underwent PCI for symptomatic coronary artery disease and DES implantation. Exclusion criteria were severe liver and kidney dysfunctions, malignant tumors, severe autoimmune diseases. Patients whose lipid levels were found to change dynamically during 1-year follow up were also excluded from the study. 


\section{Sample collection and follow-up}

Detailed medical charts were performed of the initial hospitalization to obtain patients' medical history, laboratory results, and medications. For blood lipid detection, fasting blood samples were collected from each subject and anticoagulated with ethylenediamine tetraacetic acid (EDTA) dipotassium salt in the early morning. The sample was separated immediately by centrifugation at $3000 \mathrm{~g}$ for $15 \mathrm{~min}$ at $4{ }^{\circ} \mathrm{C}$ to retrieve plasma. Lipid profiles were measured enzymatically on a chemistry analyzer (AU5400 analyzer, Beckman Coulter, CA, USA).

Follow-up were scheduled from the date of discharge for the index hospitalization. Follow-up data were collected by telephone interviews and entered into a computer database by trained staff and by inviting patients or their relatives to complete a standardized questionnaire which was sent to the patients during the followup collection period. The preferred definition of MACE was the composite of cardiovascular death, nonfatal myocardial infarction (MI), and target lesion revascularization (TLR), stent thrombosis (ST). All-cause death was defined as any death during or after the procedure and was considered to be of cardiac origin unless a definite noncardiac cause could be established. MI was defined as a recent ischaemic symptom with new electrocardiographic changes and a positive troponin concentration exceed the upper limit of normal. TLR was defined as repeated revascularization of the target vessel by PCI or surgical bypass of any vessel. ST and bleeding events were assessed according to the Bleeding Academic Research Consortium classification [24].

\section{Statistical analysis}

All statistical analyses were performed with the SPSS 19.0 statistical package program (SPSS, Chicago, IL, USA). Continuous variables were expressed as mean \pm standard deviation (SD) and categorical variables as percentage. Analysis of variance and the chi-square test were used for continuous and categorical variables, respectively. Multivariate Cox regression analysis using the entered method was used to evaluate the independent predictors of clinical endpoint. MACE event-free rates for clinical outcomes were constructed using the Kaplan-Meier method and compared with the log-rank test. A $P<0.05$ was recognized as statistically significant.

\section{Results \\ Patient characteristics}

A flow chart of the patient enrollment was provided as Fig. 1. Subsequent to coronary angiography, a total of 1968 consecutive ACS patients who underwent PCI with successful DES implantation and cholesterol-lowering therapy with atorvastatin were enrolled, of whom 1937 eligible patients were included in this analysis. Patients were allocated into low and high ratio groups according to the median value of the LDL-C/HDL-C ratio (the median value was 2.7 ) in each subgroup of study patients. The baseline clinical and demographic characteristics of the recruited patients were detailed in Table 1. The patients in low group were older than those of the high group. There was a higher proportion of ST-elevation myocardial infarction (STEMI) in low group compared to the high group. There were statistically significant differences in term of dyslipidemia, TG, LDL-C, HDL-C, hemoglobin and platelet. No significant differences were observed for other variables between the two groups.

\section{Clinical outcomes}

Table 2 shows the entire occurrence of adverse cardiovascular events according to the ratio of LDL-C/HDL-C. We found that there were no significant differences in

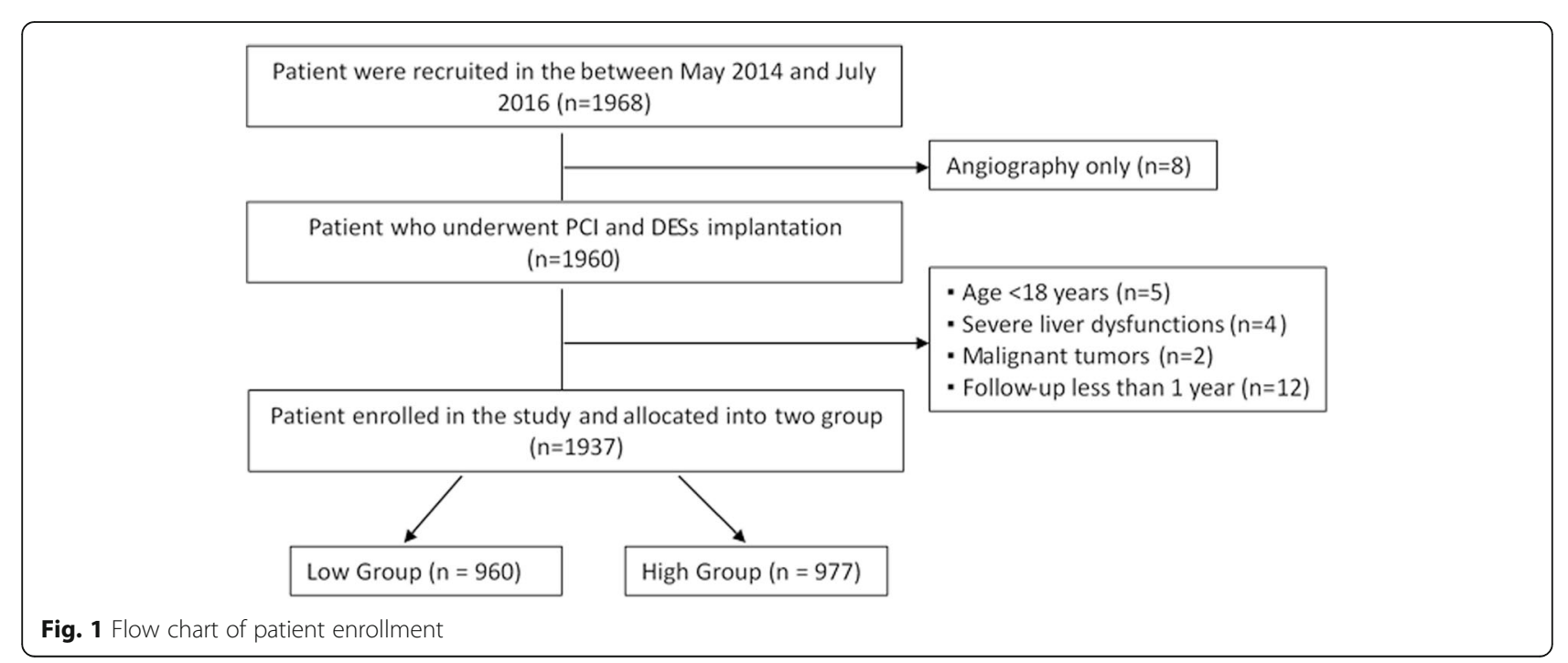


Table 1 Baseline characteristics of the study cohorts

\begin{tabular}{|c|c|c|c|c|}
\hline \multirow[t]{2}{*}{ Characteristics } & \multirow{2}{*}{$\begin{array}{l}\text { Overall } \\
(n=1937)\end{array}$} & \multirow{2}{*}{$\begin{array}{l}\text { Low } \\
(n=960)\end{array}$} & \multirow{2}{*}{$\begin{array}{l}\text { High } \\
(n=977)\end{array}$} & \multirow[t]{2}{*}{$P$ value } \\
\hline & & & & \\
\hline Age, years & $64.0 \pm 10.8$ & $65.0 \pm 10.7$ & $63.0 \pm 10.8$ & $<0.001$ \\
\hline Male, n(\%) & $1475(76.1)$ & $702(73.1)$ & $773(79.1)$ & 0.002 \\
\hline Smoking, n(\%) & $738(38.1)$ & $361(37.6)$ & $377(38.6)$ & 0.656 \\
\hline Drinking, n(\%) & $93(4.8)$ & $57(5.9)$ & $42(4.3)$ & 0.121 \\
\hline Systolic BP, mmHg & $134.0 \pm 24.6$ & $134.6 \pm 24.6$ & $133.6 \pm 24.7$ & 0.400 \\
\hline Previous MI, n(\%) & $50(2.6)$ & $30(3.1)$ & $19(1.9)$ & 0.098 \\
\hline Hypertension, n(\%) & $1066(55.0)$ & $523(54.5)$ & $543(55.6)$ & 0.627 \\
\hline Dyslipidemia, n(\%) & $704(36.3)$ & $254(26.5)$ & $450(46.1)$ & $<0.001$ \\
\hline Diabetes mellitus, n(\%) & $552(28.5)$ & $262(27.3)$ & $290(29.7)$ & 0.244 \\
\hline LV ejection fraction, (\%) & $56.3 \pm 9.6$ & $56.4 \pm 9.2$ & $56.3 \pm 10.0$ & 0.836 \\
\hline \multicolumn{5}{|l|}{ Clinical presentation } \\
\hline Unstable angina, n(\%) & $598(30.9)$ & $277(28.9)$ & $321(32.9)$ & 0.062 \\
\hline NSTEMI, n(\%) & $320(16.5)$ & $144(15.0)$ & $176(18.0)$ & 0.076 \\
\hline STEMI, n(\%) & $1022(52.8)$ & $542(56.5)$ & $480(49.1)$ & 0.001 \\
\hline \multicolumn{5}{|l|}{ Number of vessels } \\
\hline Single, $n(\%)$ & $1119(57.8)$ & $539(56.1)$ & $580(59.4)$ & 0.154 \\
\hline Double, n(\%) & $473(24.4)$ & $243(25.3)$ & $230(23.5)$ & 0.369 \\
\hline Triple, n(\%) & $345(17.8)$ & $178(18.5)$ & $167(17.1)$ & 0.407 \\
\hline Stent diameter, mm & $3.13 \pm 0.45$ & $3.13 \pm 0.44$ & $3.13 \pm 0.46$ & 0.833 \\
\hline Stent length, mm & $34.87 \pm 13.88$ & $35.45 \pm 13.22$ & $34.31 \pm 14.46$ & 0.070 \\
\hline \multicolumn{5}{|l|}{ Medications } \\
\hline Aspirin & $1737(89.7)$ & $868(90.4)$ & $869(88.9)$ & 0.297 \\
\hline Clopidogrel & $1728(89.2)$ & $868(90.4)$ & $860(88.0)$ & 0.093 \\
\hline ACEI/ARB, n(\%) & $1597(82.4)$ & $787(82.0)$ & $810(82.9)$ & 0.592 \\
\hline$\beta$-blocker, n(\%) & $1603(82.8)$ & $782(81.5)$ & $821(84.0)$ & 0.149 \\
\hline PPI, n(\%) & $1351(68.7)$ & $686(71.5)$ & $665(68.1)$ & 0.113 \\
\hline Calcium blockers, n(\%) & $269(13.9)$ & $132(13.8)$ & $137(14.0)$ & 0.862 \\
\hline \multicolumn{5}{|l|}{ Laboratory parameters } \\
\hline $\mathrm{TC}, \mathrm{mmol} / \mathrm{L}$ & $1.96 \pm 1.50$ & $1.95 \pm 1.84$ & $1.97 \pm 1.06$ & 0.751 \\
\hline $\mathrm{TG}, \mathrm{mmol} / \mathrm{L}$ & $4.94 \pm 1.23$ & $4.55 \pm 1.22$ & $5.31 \pm 1.24$ & $<0.001$ \\
\hline LDL-C, rmmol/L & $3.07 \pm 0.97$ & $2.49 \pm 0.67$ & $3.65 \pm 0.88$ & $<0.001$ \\
\hline $\mathrm{HDL}-\mathrm{C}, \mathrm{mmol} / \mathrm{L}$ & $1.18 \pm 0.42$ & $1.32 \pm 0.52$ & $1.04 \pm 0.21$ & $<0.001$ \\
\hline Hemoglobin, g/L & $134.80 \pm 27.66$ & $131.20 \pm 19.01$ & $138.30 \pm 33.73$ & $<0.001$ \\
\hline Platelet, $\times 10^{9} / \mathrm{L}$ & $232.00 \pm 75.52$ & $226.10 \pm 79.21$ & $237.80 \pm 71.28$ & $<0.001$ \\
\hline
\end{tabular}

BP Blood pressure, LV Left ventricular, NSTEMI Non-ST elevation myocardial infarction, STEMI ST elevation myocardial infarction, $L A D$ Left anterior descending artery, $L C X$ Left circumflex artery, $R C A$ Right coronary artery, $A C E I$ angiotensin-converting enzymeinhibitor, $A R B$ Angiotensin receptor blocker, $P P I$ Proton-pump inhibitor, TC Total cholesterol, TG Triglycerides, LDL-C Low-density lipoprotein cholesterol, HDL-C High-density lipoprotein cholesterol Data presented are mean \pm SD or $n(\%)$

1-year cardiovascular death (hazard ratio [HR]: 1.97 , 95\% confidence interval [CI]: 0.49 to $7.84, P=0.329)$, MI (HR: $1.66,95 \% \mathrm{CI}: 0.84$ to $3.28, P=0.172$ ) and bleeding events (HR: $1.08,95 \% \mathrm{CI}: 0.83$ to $1.41, P=0.598)$. The cumulative incidence of TLR (HR: $1.43,95 \% \mathrm{CI}: 1.10$ to 1.86 , $P=0.007$ ), ST (HR: 2.04, 95\% CI: 1.06 to $3.93, P=0.0368$ ) and MACE (HR: $1.54,95 \% \mathrm{CI}: 1.24$ to $1.91, P<0.001$ ) were significantly higher in high group than in low group. Multivariate Cox regression analysis of the association between the MACE and multiple parameters are presented in Table 3. The results of the multivariable Cox proportional hazards model demonstrated that age (HR: 1.556, 95\%, CI:1.198 to 2.021, $P<0.001$ ), together with diabetes mellitus (HR: 1.490, 95\% CI:1.142 to 
Table 2 Clinical outcome up to 1-year

\begin{tabular}{|c|c|c|c|c|}
\hline & Low $(n=960)$ & High $(n=977)$ & Hazard ratio $(95 \%$ Cl) & $P$ value \\
\hline Death & $5(0.52)$ & $8(0.82)$ & 1.57 (0.52 to 4.79$)$ & 0.580 \\
\hline Cardiovascular death & $3(0.31)$ & $6(0.61)$ & 1.97 (0.49 to 7.84$)$ & 0.329 \\
\hline Myocardial infarction & $13(1.35)$ & $22(2.25)$ & 1.66 (0.84 to 3.28$)$ & 0.172 \\
\hline STEMI & $8(0.83)$ & 19 (1.94) & 2.33 (1.03 to 5.31$)$ & 0.051 \\
\hline NSTEMI & $3(0.31)$ & $3(0.31)$ & 0.98 (0.20 to 4.86$)$ & 1.000 \\
\hline TLR & $85(8.86)$ & $124(12.69)$ & $1.43(1.10$ to 1.86$)$ & 0.007 \\
\hline Stent thrombosis & $13(1.35)$ & $27(2.76)$ & 2.04 (1.06 to 3.93 ) & 0.037 \\
\hline MACE & $114(11.88)$ & 179 (18.32) & 1.54 (1.24 to 1.91$)$ & $<0.001$ \\
\hline Bleeding events & $93(9.69)$ & $102(10.44)$ & $1.08(0.83$ to 1.41$)$ & 0.598 \\
\hline
\end{tabular}

CI Confidence interval, NSTEMI Non-ST elevation myocardial infarction, STEMI ST elevation myocardial infarction, TLR Target lesion revascularization, MACE Major adverse cardiac events; Other abbreviations as in Table 1

Data are presented as $\mathrm{n}(\%)$

1.945, $P=0.003$ ), and ratio of LDL-C/HDL-C (HR: 1.638, 95\% CI:1.260 to $2.218, P<0.001)$ were independent predictors of 1-year MACE. Detailed results of the multivariable analysis were shown in Table 3. The Kaplan-Meier cumulative MACE-free survival curves with a log-rank test showed that the presence of high ratio of LDL-C/ HDL-C was associated with higher incidences of MACE after PCI with DES implantation (Fig. 2).

\section{Discussion}

To the best of our knowledge, this is the first study reporting regards the associations of LDL-C/HDL-C ratio with the incidences of 1 -year adverse outcomes of ACS patients treated with atorvastatin after PCI and DES implantation in Chinese population. In the present study, we found that the rate of TLR, ST and MACE in patients with the high LDL-C/HDL-C ratio was significantly greater than those with the low LDL-C/HDL-C ratio at 1-year follow-up. The result of multivariate analysis showed that older age, diabetes mellitus and the high ratio of LDL-C/HDL-C factors were positively associated with the accumulated MACE during 1-year follow-up. The results presented here indicate that of LDL-C/HDL-C ratio was predictor of MACE at one year in patients with ACS who underwent PCI and DES.
ACS is one of leading cause of death in developed and developing countries. PCI and the application of stents resulted in tremendous progress on the management of patients with ACS, which dramatically reduces the cardiovascular mortality and disability rates $[4,6]$. ST and other adverse cardiovascular events, including death, MI, TLR and bleeding events, are considered as lifethreatening complications of PCI [25]. Dual antiplatelet therapy consisting of aspirin and clopidogrel is the standard therapy choice for patients with ACS after PCI. However, some patients on standard dual antiplatelet therapy still have at risk of adverse cardiovascular events after DES implantation due to the existence of high platelet reactivity, genetic variation with drug response, older age and dyslipidemia $[7,8,13]$.

Numerous of evidences have already revealed that LDL-C serum concentrations is associated with cardiovascular risk and intensive treatment with statin is recommended to reduce the rate of recurrent ischaemic events and stent thrombosis in patients with ACS [13, 26]. Every $1.0 \mathrm{mmoL} / \mathrm{L}$ reduction in LDL-C is associated with a corresponding $20-25 \%$ reduction in cardiovascular mortality and non-fatal myocardial infarction. According to the current European guidelines, as well as the US guidelines, a treatment goal of $\mathrm{LDL}-\mathrm{C}<70 \mathrm{mg} / \mathrm{dL}$ is recommended $[16,17]$. In spite of the emphasis of guidelines on

Table 3 Results of a multivariable Cox proportional hazards model

\begin{tabular}{lll}
\hline Variable & Hazard ratio (95\% Cl) & $P$ value \\
\hline Age (age $\geq 65$ vs $<65)$ & $1.556(1.198$ to 2.021$)$ & 0.001 \\
Gender (male vs female) & $0.958(0.696$ to 1.317$)$ & 0.790 \\
Smoking (active smoker vs non-smoker) & $0.813(0.603$ to 1.098$)$ & 0.177 \\
Drinking (drinker vs non-drinker) & $0.944(0.486$ to 1.833$)$ & 0.865 \\
Hypertension (hypertensive vs normotensive) & $0.997(0.771$ to 1.289$)$ & 0.981 \\
Diabetes mellitus (diabetic vs non-diabetic) & $1.490(1.142$ to 1.945$)$ & 0.003 \\
LDL-C/HDL-C (Ratio $\geq 2.7$ vs $<2.7)$ & $1.638(1.260$ to 2.128$)$ & $<0.001$ \\
\hline
\end{tabular}




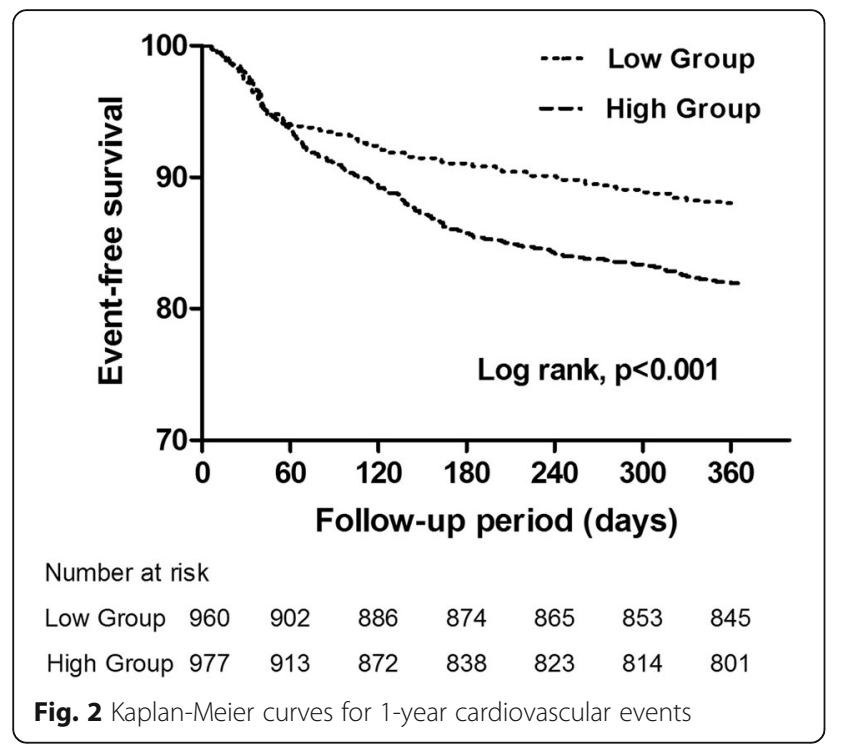

the tight control of the LDL-C level, several surveys have shown that still a large number of patients remain undertreated and do not attain LDL-C treatment goals $[11,12,27]$. Similarly, our result is in line with that reported in previous studies.

It is also important to note that mixed dyslipidaemia played an important role in the propagation of coronary artery disease [28]. Epidemiological data have demonstrated that low levels of high-density lipoprotein cholesterol (HDL-C) was an important risk factor for progression of coronary atherosclerosis and moderate increases in HDL-C in statin-treated patients are correlated with regression of coronary atherosclerosis $[20,29,30]$. It has been also suggested that HDL-C could reduce the risk and extent of PCI-related myocardial infarction by stabilizing plaques. However, a paradoxical decrease in HDL-C levels after statin therapy is often seen in clinical settings. Previous lipidlowering trial have shown that the stable lipid level after statin treatment is achieved after 4-6 weeks, and then the lowered lipid level remained during the subsequent maintenance treatment $[26,31]$. Take these things together, the ratio of LDL-C/HDL-C ratio might be the useful indicators for prognosis prediction as it simultaneously evaluates the levels of both LDL-C and HDL-C. Some recent studies have considered the LDL-C/HDL-C ratio to be a sensitive marker of the risk of atherosclerotic cardiovascular disease, as it simultaneously evaluates the levels of both LDL-C and HDL-C [32, 33]. Several studies have previously demonstrated the LDL-C/HDL-C ratio is an important contributor to the development of new coronary artery disease in patients with a previous history of PCI [22]. Moreover, a prior report has found that the LDL-C/HDL-C ratios is a useful indicator for preventing the progression of atherosclerotic cardiovascular disease
[34]. In the present study, our results indicated that the high LDL-C/HDL-C ratio group was at a high risk of 1-year adverse outcomes in patients with ACS after PCI and DES implantation. In addition, several factors including advanced age, diabetes mellitus and major organ dysfunction are well-established predictors of worse clinical outcomes in patients with ACS or PCI $[9,35,36]$. In current study, the multivariable Cox hazards analysis of MACE in our study revealed that older age, diabetes mellitus and the high ratio of LDL-C/HDL-C were independent predictors for the MACE at 1-year follow up. Moreover, the Kaplan-Meier cumulative MACE-free survival curves with a log-rank test also showed that the presence of high ratio of LDL-C/HDL-C might serve as a predictor of one year clinical outcomes in patients with ACS after PCI and DES implantation. Our results are consistent with the findings from previous study.

Our study has several limitations. First, a major limitation is its retrospective design and was subject to bias. Second, as this is a single center study and the sample size was relatively small, the evidence may not be as persuasive as that obtained by a larger scale and multiple regions. Third, the follow-up period was relatively short, a long-term prospective large-scale study is needed.

\section{Conclusions}

In conclusion, the results of this study found that the high LDL-C/HDL-C ratio was associated with cardiovascular events in patients with ACS after PCI and DES implantation. For patients with ACS after DES implantation, LDL-C/HDL-C ratio may help in planning more aggressive cholesterol-lowering therapy to improve the clinical outcome. Further large prospective studies are needed to confirm and to reveal clinical implications of our findings.

\section{Abbreviations}

ACEl: Angiotensin-converting enzymeinhibitor; ACS: Acute coronary syndromes; AMI: Acute myocardial infarction; ARB: Angiotensin receptor blocker; BP: Blood pressure; CABG: Coronary artery bypass graft;

CAD: Cardiovascular diseases; Cl: Confidence interval; DES: Drug-eluting stents; EDTA: Ethylenediamine tetraacetic acid; HDL-C: High-density lipoprotein cholesterol; HR: Hazard ratio; LAD: Left anterior descending artery; LCX: Left circumflex artery; LDL-C: Low-density lipoprotein cholesterol; LV: Left ventricular; MACE: Major adverse cardiac events; NSTEMI: Non-ST elevation myocardial infarction; PCl: Percutaneous coronary intervention; PPI: Proton-pump inhibitor; RCA: Right coronary artery; SD: Standard deviation; ST: Stent thrombosis; STEMI: ST elevation myocardial infarction; TC: Total cholesterol; TG: Triglycerides; TLR: Target lesion revascularization

\section{Acknowledgments}

The author would like to thank other colleagues whom were not listed in the authorship of Clinical Core Laboratory and Clinical Core Laboratory, Center for Precision Medicine, Guangdong Provincial Engineering and Technology Research Center for Clinical Molecular Diagnostics and Antibody Therapeutics, Guangdong Provincial Key Laboratory of Precision Medicine and Clinical Translational Research of Hakka Population, Meizhou People's Hospital (Huangtang Hospital), Meizhou Academy of Medical Sciences, Meizhou Hospital Affiliated to Sun Yat-sen University for their helpful comments on the manuscript. 


\section{Funding}

This study was supported by The National Key Research and Development Program of China (Grant No.: 2017 YFD0501705 to Dr. Pingsen Zhao), Natural Science Foundation of Guangdong Province, China (Grant No.:

2016A030307031 to Dr. Pingsen Zhao), The National Key Research and Development Program of China (Grant No.: 2016YFD0500405 to Dr. Pingsen Zhao), Medical Scientific Research Foundation of Guangdong Province, China (Grant No.: A2016306 to Dr. Pingsen Zhao) and Key Scientific and Technological Project of Meizhou People's Hospital (Huangtang Hospital), Meizhou Academy of Medical Sciences, Meizhou Hospital Affiliated to Sun Yat-sen University, Guangdong Province, China (Grant No.: MPHKSTP20170102 to Dr. Pingsen Zhao), and Medical Scientific Research Foundation of Guangdong Province, China (Grant No.: A2017404 to Dr. Jingyuan Hou).

\section{Availability of data and materials}

All data generated or analysed during this study are included in this published article.

\section{Authors' contributions}

PZ conceived and designed the experiments; $\mathrm{ZZ}, \mathrm{JH}$ and PZ recruited subjects and collected clinical data. JH conducted the laboratory testing. QZ, WZ, BL, CL, ZL and MY helped to analyze the data. PZ, ZZ and JH prepare the manuscript. PZ and ZZ reviewed the manuscript. All authors read and approved the final manuscript.

\section{Ethics approval and consent to participate} Not applicable.

\section{Consent for publication}

Not applicable.

\section{Competing interests}

The authors declare that they have no competing interests.

\section{Publisher's Note}

Springer Nature remains neutral with regard to jurisdictional claims in published maps and institutional affiliations.

\section{Author details}

${ }^{1}$ Center for Cardiovascular Diseases, Meizhou People's Hospital (Huangtang Hospital), Meizhou Academy of Medical Sciences, Meizhou Hospital Affiliated to Sun Yat-sen University, Meizhou 514031, People's Republic of China. ${ }^{2}$ Clinical Core Laboratory, Meizhou People's Hospital (Huangtang Hospital), Meizhou Academy of Medical Sciences, Meizhou Hospital Affiliated to Sun Yat-sen University, Meizhou 514031, People's Republic of China. ${ }^{3}$ Center for Precision Medicine, Meizhou People's Hospital (Huangtang Hospital), Meizhou Academy of Medical Sciences, Meizhou Hospital Affiliated to Sun Yat-sen University, Meizhou 514031, People's Republic of China. ${ }^{4}$ Guangdong Provincial Engineering and Technological Research Center for Molecular Diagnostics of Cardiovascular Diseases, Meizhou 514031, People's Republic of China. ${ }^{5}$ Meizhou Municipal Engineering and Technology Research Center for Molecular Diagnostics of Cardiovascular Diseases, Meizhou 514031, People's Republic of China. ${ }^{6}$ Meizhou Municipal Engineering and Technology Research Center for Molecular Diagnostics of Major Genetic Disorders, Meizhou 514031, People's Republic of China. 'Guangdong Provincial Key Laboratory of Precision Medicine and Clinical Translational Research of Hakka Population, Meizhou 514031, People's Republic of China. ${ }^{8}$ Guangdong Provincial Engineering and Technology Research Center for Clinical Molecular Diagnostics and Antibody Therapeutics, No 63 Huangtang Road, Meijiang District, Meizhou 514031, People's Republic of China.

Received: 1 August 2018 Accepted: 21 January 2019 Published online: 02 February 2019

\section{References}

1. Li H, Ge J. Global, regional, and national age-sex specific all-cause and cause-specific mortality for 240 causes of death, 1990-2013: a systematic analysis for the Global Burden of Disease Study 2013. Lancet. 2015;385:117171.

2. Li H, Ge J. Cardiovascular diseases in China: current status and future perspectives. Int J Cardiol Heart Vasc. 2015;6:25-31.
3. Windecker S, Kolh P, Alfonso F, Collet JP, Cremer J, Falk V, et al. ESC/EACTS guidelines on myocardial revascularization: the task force on myocardial revascularization of the European Society of Cardiology (ESC) and the European Association for Cardio-Thoracic Surgery (EACTS) developed with the special contribution of the European Association of Percutaneous Cardiovascular Interventions (EAPCI). Eur Heart J. 2014;2014(35):2541-619.

4. Naito R, Miyauchi K, Konishi H, Tsuboi S, Ogita M, Dohi T, et al. Comparing mortality between coronary artery bypass grafting and percutaneous coronary intervention with drug-eluting stents in elderly with diabetes and multivessel coronary disease. Heart Vessel. 2016;31:1424-9.

5. Laskey WK, Yancy CW, Maisel WH. Thrombosis in coronary drug-eluting stents: report from the meeting of the circulatory system medical devices advisory panel of the Food and Drug Administration Center for devices and radiologic health, December 7-8. 2006. Circulation. 2007;115:2352-7.

6. Babapulle MN, Joseph L, Belisle P, Brophy JM, Eisenberg MJ. A hierarchical Bayesian meta-analysis of randomised clinical trials of drug-eluting stents. Lancet. 2004;364:583-91.

7. Kaneko H, Yajima J, Oikawa Y, Tanaka S, Fukamachi D, Suzuki S, et al. Impact of aging on the clinical outcomes of Japanese patients with coronary artery disease after percutaneous coronary intervention. Heart Vessel. 2014;29:156-64.

8. Simon T, Verstuyft C, Mary-Krause M, Quteineh L, Drouet E, Meneveau N, et al. Genetic determinants of response to clopidogrel and cardiovascular events. N Engl J Med. 2009;360:363-75.

9. Beckman JA, Paneni F, Cosentino F, Creager MA. Diabetes and vascular disease: pathophysiology, clinical consequences, and medical therapy: part II. Eur Heart J. 2013;34:2444-52.

10. Hong SJ, Kim MH, Ahn TH, Ahn YK, Bae JH, Shim WJ, et al. Multiple predictors of coronary restenosis after drug-eluting stent implantation in patients with diabetes. Heart. 2006;92:1119-24.

11. Ogita M, Miyauchi K, Miyazaki T, Naito R, Konishi H, Tsuboi S, et al. Low high-density lipoprotein cholesterol is a residual risk factor associated with long-term clinical outcomes in diabetic patients with stable coronary artery disease who achieve optimal control of low-density lipoprotein cholesterol. Heart Vessel. 2014;29:35-41.

12. Landes U, Kornowski R, Assali A, Vaknin-Assa H, Greenberg G, Lev El, et al. Predictors of long term outcomes in 11,441 consecutive patients following percutaneous coronary interventions. Am J Cardiol. 2015;115:855-9.

13. Arsenault BJ, Rana JS, Stroes ES, Despres JP, Shah PK, Kastelein JJ, et al. Beyond low-density lipoprotein cholesterol respective contributions of non-highdensity lipoprotein cholesterol levels, triglycerides, and the total cholesterol/ high-density lipoprotein cholesterol ratio to coronary heart disease risk in apparently healthy men and women. J Am Coll Cardiol. 2009;55:35-41.

14. Hosseini SK, Tahvildari M, Alemzadeh Ansari MJ, Nakhjavani M, Esteghamati A, Lotfi Tokaldany M. Clinical lipid control success rate before and after percutaneous coronary intervention in Iran; a single center study. Iran Red Crescent Med J. 2013;15:467-72.

15. Zhong Z, Liu J, Li B, Li C, Liu Z, Yang M, et al. Serum lipid profiles in patients with acute myocardial infarction in Hakka population in southern China. Lipids Health Dis. 2017;16:246.

16. Wada H, Ogita M, Miyauchi K, Shitara J, Endo H, Doi S, et al. Impact of gender difference on long-term outcomes of percutaneous coronary intervention for coronary artery disease in patients under statin treatment. Heart Vessel. 2017:32:16-21.

17. Laufs U, Karmann B, Pittrow D. Atorvastatin treatment and LDL cholesterol target attainment in patients at very high cardiovascular risk. Clin Res Cardiol. 2016;105:783-90.

18. Zhong Z, Liu J, Zhang Q, Zhong W, Li B, Li C, et al. Relationship between preoperative low-density lipoprotein cholesterol and Periprocedural myocardial injury in patients following elective percutaneous coronary intervention in southern China. Med Sci Monit. 2018;24:4154-61.

19. Baigent C, Blackwell L, Emberson J, Holland LE, Reith C, Bhala N, et al. Efficacy and safety of more intensive lowering of LDL cholesterol: a meta-analysis of data from 170,000 participants in 26 randomised trials. Lancet. 2010;376:1670-81.

20. Sattler KJ, Herrmann J, Yun S, Lehmann N, Wang Z, Heusch G, et al. High high-density lipoprotein-cholesterol reduces risk and extent of percutaneous coronary intervention-related myocardial infarction and improves long-term outcome in patients undergoing elective percutaneous coronary intervention. Eur Heart J. 2009;30:1894-902.

21. Seo SM, Choo EH, Koh YS, Park MW, Shin DI, Choi YS, et al. High-density lipoprotein cholesterol as a predictor of clinical outcomes in patients 
achieving low-density lipoprotein cholesterol targets with statins after percutaneous coronary intervention. Heart. 2011;97:1943-50.

22. Endo A, Yoshida Y, Kageshima K, Sato H, Suga T, Nasu H, et al. Contributors to newly developed coronary artery disease in patients with a previous history of percutaneous coronary intervention beyond the early phase of restenosis. Intern Med. 2014;53:819-28.

23. Matsumoto I, Miyake Y, Mizukawa M, Takagi Y. Impact of low-density lipoprotein cholesterol/high-density lipoprotein cholesterol ratio on longterm outcome in patients undergoing percutaneous coronary intervention. Circ J. 2011;75:905-10.

24. Ndrepepa G, Schuster T, Hadamitzky M, Byrne RA, Mehilli J, Neumann FJ, et al. Validation of the bleeding academic research consortium definition of bleeding in patients with coronary artery disease undergoing percutaneous coronary intervention. Circulation. 2012;125:1424-31.

25. Sibbing D, Stegherr J, Latz W, Koch W, Mehilli J, Dorrler K, et al. Cytochrome P450 2C19 loss-of-function polymorphism and stent thrombosis following percutaneous coronary intervention. Eur Heart J. 2009:30:916-22.

26. Cannon CP, Braunwald E, McCabe CH, Rader DJ, Rouleau JL, Belder R, et al. Intensive versus moderate lipid lowering with statins after acute coronary syndromes. N Engl J Med. 2004;350:1495-504.

27. Miyauchi K, Ray K. A review of statin use in patients with acute coronary syndrome in Western and Japanese populations. J Int Med Res. 2013;41: 523-36.

28. Stoll G, Bendszus M. Inflammation and atherosclerosis: novel insights into plaque formation and destabilization. Stroke. 2006;37:1923-32.

29. Nissen SE, Nicholls SJ, Sipahi I, Libby P, Raichlen JS, Ballantyne CM, et al. Effect of very high-intensity statin therapy on regression of coronary atherosclerosis: the ASTEROID trial. JAMA. 2006;295:1556-65.

30. Li XL, Guo YL, Zhu CG, Xu RX, Qing P, Wu NQ, et al. Relationship of highdensity lipoprotein cholesterol with periprocedural myocardial injury following elective percutaneous coronary intervention in patients with low-density lipoprotein cholesterol below 70 mg/dL. J Am Heart Assoc. 2015;4:e001412.

31. Yun KH, Oh SK, Rhee SJ, Yoo NJ, Kim NH, Jeong JW. 12-month follow-up results of high dose rosuvastatin loading before percutaneous coronary intervention in patients with acute coronary syndrome. Int J Cardiol. 2011;146:68-72.

32. Barter P, Gotto AM, LaRosa JC, Maroni J, Szarek M, Grundy SM, et al. HDL cholesterol, very low levels of LDL cholesterol, and cardiovascular events. N Engl J Med. 2007:357:1301-10.

33. Kurebayashi N, Yoshikawa D, Ishii H, Sato B, Ando H, Okada T, et al. Impact of the low- to high-density lipoprotein cholesterol ratio on composition of angiographically ambiguous left main coronary artery plaque. Circ J. 2011;75:1960-7.

34. Nicholls SJ, Tuzcu EM, Sipahi I, Grasso AW, Schoenhagen P, Hu T, et al. Statins, high-density lipoprotein cholesterol, and regression of coronary atherosclerosis. JAMA. 2007:297:499-508.

35. Goldfine AB. Assessing the cardiovascular safety of diabetes therapies. N Engl J Med. 2008;359:1092-5.

36. Donahoe SM, Stewart GC, McCabe CH, Mohanavelu S, Murphy SA, Cannon CP, et al. Diabetes and mortality following acute coronary syndromes. JAMA. 2007;298:765-75.

Ready to submit your research? Choose BMC and benefit from:

- fast, convenient online submission

- thorough peer review by experienced researchers in your field

- rapid publication on acceptance

- support for research data, including large and complex data types

- gold Open Access which fosters wider collaboration and increased citations

- maximum visibility for your research: over $100 \mathrm{M}$ website views per year

At $\mathrm{BMC}$, research is always in progress.

Learn more biomedcentral.com/submissions 